\title{
Setting the ground rules: Use and practice of ground rules in child forensic interviews
}

\author{
Melanie B. Fessinger, M.L.S. ${ }^{1,2}$ \\ Kelly McWilliams, Ph.D. ${ }^{2}$ \\ Faizun N. Bakth ${ }^{2}$ \\ Thomas D. Lyon, J.D., Ph.D. ${ }^{3}$ \\ ${ }^{1}$ Graduate Center, City University of New York \\ ${ }^{2}$ John Jay College of Criminal Justice, City University of New York \\ ${ }^{3}$ University of Southern California
}

In press, Child Maltreatment

\begin{abstract}
Author Notes
This research was supported by Eunice Kennedy Shriver National Institute of Child Health and Human Development Grant HD087685 to Thomas D. Lyon. The first author was supported by the National Science Foundation Graduate Research Fellowship Program under Grant No. 1610400 during the preparation of this manuscript. The content of this manuscript is solely the responsibility of the authors and does not represent the official views of either funding agency. Correspondence concerning this article should be addressed to Kelly McWilliams, Department of Psychology, John Jay College of Criminal Justice, 524 W. 59th Street, New York, NY, 10019, kmcwilliams@jjay.cuny.edu.
\end{abstract}




\begin{abstract}
Most child forensic interviewing protocols recommend that interviewers administer a series of ground rules to emphasize concepts that are important to accurately answering interview questions. Limited research has examined whether interviewers follow ground rules recommendations in real-world forensic interviews. In this study, we examined how often highly trained interviewers presented and practiced each of the recommended ground rules. We also examined whether children accurately responded to practice questions. We coded transcripts from 241 forensic interviews of 4- to 12-year-old children conducted by interviewers in the United States who were largely trained using the Ten Step Investigative Interview (Lyon, 2014). Results demonstrated that interviewers routinely presented and practiced the ground rules, but this significantly varied by children's age. Additionally, children often accurately responded to practice questions, but younger children were less accurate than older children. Taken together, results highlight that interviewers may deviate from ground rules recommendations based on the characteristics of the child which has implications for both future research and practice.
\end{abstract}




\section{Setting the ground rules: Use and practice of ground rules in child forensic interviews}

Most child forensic interviewing protocols recommend that interviewers present children with a series of ground rules to emphasize concepts that are important to accurately answering interview questions, to clarify the conversational style, and to set expectations for children's behavior (ASPAC Taskforce, 2012; Lyon, 2014; Orbach, Hershkowitz, Lamb, Esplin, \& Horowitz, 2000). Additionally, most protocols recommend that interviewers practice several of the ground rules to allow children an opportunity to apply the concepts inherent to the rules. The topics and exact wording of ground rules vary across protocols, but they typically encourage children to say "I don't know" rather than guess ("I don't know"), to ask for clarification when they do not understand a question (“I don't understand"), to correct any mistakes the interviewer makes ("you're wrong"), to understand that the interviewer does not share their knowledge ("ignorant interviewer"), and to tell the truth ("promise to tell the truth").

Limited research has examined the use of ground rules. The research that is available has yielded inconsistent results (see Brubacher, Poole, \& Dickinson, 2015 for a review). In essence, ground rules may have benefits that are dependent on children's age, the specific rule, the style of administration (i.e., presentation versus practice), the combination of rules, and the definition of "benefits."

Although most protocols outline a specific method for administering ground rules, deviations or adjustments from those methods may produce different results. For example, one study examining children's responses to unanswerable questions found that children's age and the specific combination of ground rules affected children's willingness to say "I don't know" (Waterman \& Blades, 2011). For six year olds, combining the I don't know, I don't understand, and ignorant interviewer ground rules significantly increased children's appropriate responses to 
unanswerable questions (e.g., saying "I don't know") compared to omitting the ignorant interviewer ground rule. However, the combination of instructions did not affect eight-year-olds responding behavior. This finding demonstrates the possibility that deviations from empiricallybased recommendations can result in significant changes in children's responses. Therefore, it is important to know whether actual forensic interviewers deviate from the protocols and to empirically examine the effect of those deviations.

Interviewers may systematically deviate from the protocols or deviate based on the needs of each child they interview. In their most recent recommendations, the National Children's Advocacy Center (NCAC) suggested that " $[\mathrm{t}]$ he process of providing rules may be confusing or overwhelming to a young preschooler or developmentally delayed child. In such cases, [the ground rules] step may be eliminated or adapted to the perceived needs of the child" (NCAC, 2019, p. 10). Accordingly, it may be the case that interviewers following this recommendation may omit the ground rules altogether with particularly young children. This recommendation highlights one area of ground rules administration where actual practice and empirically-based recommendations may vary. Identifying these and other areas where practice and empiricallybased recommendations differ provides important insight on areas of future study. If interviewers are adjusting their practices or omitting parts of an interview based on their own assessments of children's abilities, these strategies need to be identified and tested to understand their effects on children's responding behavior.

\section{Use of Ground Rules in Real-World Contexts}

Field studies provide important insight into how ground rules are used in real-world contexts. Yet, only a handful of studies have examined the use of ground rules in real legal settings (Ahern, Stolzenberg \& Lyon, 2015; Earhart, Brubacher, Powell, Westera, \& Goodman- 
Delahunty, 2017; Earhart, La Rooy, Brubacher, \& Lamb, 2014; Teoh \& Lamb, 2010). In one study, Teoh and Lamb (2010) assessed 75 interviews using the NICHD protocol with children alleging maltreatment in Malaysia. Interviewers in their sample used a higher proportion of their pre-substantive prompts discussing instructions with older children (13- to 15-year-olds) than younger children (5- to 7-year-olds). Only among young children (5- to 7- year-olds), a higher proportion of pre-substantive prompts used for instructions was positively related to children's informative responses given during the substantive phase of the interview. Thus, young children may have benefited from the instructions even though interviewers were less likely to present them to their age group. In another study, Earhart and colleagues (2014) assessed a subsample of 76 interviews using the Memorandum of Good Practice protocol. Interviewers in their sample almost always presented the promise to tell the truth ground rule, less often presented I don't know, and rarely presented ignorant interviewer. Interestingly, use of the I don't know instruction was not related to children's "I don't know" responses during the interview. Together, these studies illustrate that spending more time presenting the ground rules might increase children's informative responding, but the exact mechanism driving this pattern is unclear given that the mere presence of one ground rule (i.e., I don't know) did not directly affect that responding behavior (i.e., "I don't know" responses). Moreover, these studies provide preliminary evidence that interviewers may systematically deviate in their ground rule administration based on children's age and the specific ground rule in question.

Children are more likely to apply certain ground rules when they are given the opportunity to practice (Danby, Brubacher, Sharman, \& Powell, 2015). Practice is important because it gives children a chance to demonstrate their understanding of the ground rules and it gives interviewers a chance to assess children's understanding and provide any necessary 
feedback. However, children are seldom given the opportunity to practice applying the ground rules. In Earhart et al. (2014), despite half of the interviewers presenting the I don't know ground rule, no interviewers allowed children to practice applying it after presenting the instruction. Other legal contexts see similar exclusion of practice. Prosecutors and judges seldom present and even less often practice ground rules with children in courtroom settings (Ahern et al., 2015; Earhart et al., 2017). Although children tend to accurately respond to practice questions, younger children struggle more with the concepts than older children (Dickinson, Brubacher, \& Poole, 2015). For example, one laboratory study found that the majority of 4-year-old children incorrectly responded to practice questions for the I don't understand instruction (Dickinson et al., 2015). Thus, omitting the ground rules practice can lead to a missed opportunity to provide feedback to children who do not understand the rules. Yet, only one of the aforementioned studies (Earhart et al., 2014) examined practice (or lack thereof) of the ground rules in forensic interviews. Whether this pattern of omission generalizes to interviewers using different protocols needs to be examined. Additionally, to our knowledge, no field studies have examined children's accuracy in responding to practice questions when presented, which is an important component to understanding how ground rules administration functions in real-world contexts.

\section{Overview}

We conducted the present study to extend past research and to answer the call for more research on ground rules in forensic interviews. The present study examined three questions: (i) how many of the recommended ground rules (I don't know, I don't understand, you're wrong, ignorant interviewer, and promise to tell the truth) interviewers presented during their interviews; (ii) how many of the recommended ground rules (I don't know, I don't understand, you're wrong) interviewers practiced during their interviews; and (iii) how children performed 
on practice questions. Additionally, for all three questions, we were interested in whether any observed patterns varied as a function of children's age or by the specific ground rule.

Hypotheses. First, we expected that interviewers would often present each of the five recommended ground rules as consistent with their protocol. However, for reasons similar to those cited in the most recent NCAC recommendations (NCAC, 2019), we expected that interviewers would present the ground rules less often to younger children than older children. Second, we expected that interviewers would often practice each of the three recommended ground rules as consistent with their protocol. However, we expected that they would practice more with younger children than older children because younger children might need to be oriented to the conversational expectations of an interview more than older children. Third, as consistent with past laboratory research (Dickinson et al., 2015), we expected children would accurately respond to most practice questions, but that younger children would be less accurate than older children.

\section{Method}

\section{Sample}

We reviewed transcripts from 241 forensic interviews of 4- to 12-year-old children $(M=$ $7.77, S D=2.65 ; 77.18 \%$ female) alleging sexual abuse. Interviews were conducted in California between 2004 and 2015 at five different interview sites. Interviews were conducted in children's preferred language and only those conducted in English (without a translator) were included in the sample. Most of the interviewers would have received the California Forensic Interview Training (CFIT), a state-wide program that provides interviewers the Ten Step Investigative Interview protocol (Lyon, 2014). Allegations were most often against parental figures (36.51\%), followed by other family members (33.61\%), familiar persons (e.g., neighbor, coach, 28.63\%), 
and rarely against strangers (1.24\%). Slightly over half of children (58.92\%) alleged "lower severity" abuse (e.g., fondling); the remaining children (41.07\%) alleged "higher severity" abuse (e.g., penile-vaginal penetration, oral copulation).

\section{Coding}

Two independent research assistants read and coded twenty percent of the interviews. A senior author, who did not conduct any coding and was blind to interview and child characteristics, resolved any discrepancies. Interrater reliability between the research assistants was acceptably high (Cohen's Kappas $>.85)$. After reaching acceptable reliability, one of the research assistants coded the remainder of the transcripts.

Ground rules presentation. We coded whether interviewers presented some form of each of the five different ground rules recommended in the Ten Step protocol (i.e., I don't know, I don't understand, you're wrong, ignorant interviewer, promise to tell the truth). We coded the presence of each specific ground rule separately (absent, present). We then calculated a total score of ground rules presented by summing the number of ground rules that were present within each interview which resulted in a possible range of scores from 0 - 5.

Ground rules practice. We also coded whether interviewers practiced each of the three ground rules recommended in the Ten Step protocol (i.e., I don't know, I don't understand, you're wrong). The Ten Step protocol does not include practice for the ignorant interviewer and promise to tell the truth ground rules. Practice examples included "What is my dog's name?" (I don't know), "What is your ocular pigmentation?" (I don't understand), and "If I say you are thirty years old, what would you say?" (you're wrong). We coded the practice of each specific ground rule separately (no practice, practice). We then calculated a total score of ground rules 
practiced by summing the number of ground rules that were practiced within each interview which resulted in a possible range of scores from $0-3$.

Ground rules performance. We also coded whether children accurately responded to practice questions. We considered children's responses accurate when they answered with some version of the encouraged response (e.g., said "I don't know" to the I don't know practice "what is my dog's name?") and inaccurate when they answered without the encouraged response (e.g., guessed to the I don't know practice). We coded their accuracy to each practice question separately (inaccurate, accurate). We then calculated a total accuracy proportion score across ground rules by dividing the number of accurate responses over the total number of practice questions that the child received which resulted in a possible range of scores from 0 - 100 .

\section{Results}

Preliminary analyses revealed that interviewers' use of ground rules was not related to child gender, case characteristics, or interview location; therefore, these variables were not considered further. ${ }^{1}$

\section{Ground Rules Presentation}

Interviewers presented an average of $4.00(S D=1.38)$ out of 5 ground rules per interview. The number of ground rules they presented significantly differed as a function of children's age, $F(2,240)=13.92, p<.001, \eta^{2}=.11$. Post-hoc Fisher's LSD tests demonstrated that the 4- to 6-year-olds received significantly fewer ground rules $(M=3.42, S D=1.63)$ than

\footnotetext{
${ }^{1}$ We also examined whether interviewers' use of ground rules was related to interview quality. We calculated interview quality creating a proportion of optimal question types (including invitations such as "tell me" and directives such as who, what, where, and why) compared to the total number of questions in the interview. Interviewers' use of ground rules was related to interview quality; interviewers with a higher proportion of optimal questions during their interview were more likely to have presented each of the ground rules with children $(r$ 's $>.16$, $p$ 's $<.01)$ and more likely to have practiced each of the ground rules with children $(r$ 's $>.19, p$ 's $<.01)$ with the exception of practicing the you're wrong ground rule which was marginally significant, $r(207)=.12, p=.075$.
} 
the 7- to 9 -year-olds $(M=4.34, S D=1.13 ; p<.001)$ and the 10 - to 12 -year-olds $(M=4.34, S D=$ $1.04 ; p<.001)$. The two older age groups did not differ from one another $(p=.97)$.

We then conducted follow-up analyses to examine the age effect across the five ground rules. We conducted separate logistic regressions with age as the predictor (using the youngest age as the comparison group) and the presence of each ground rule as the criterion. Full results are presented in Table 1 and Figure 1. Age significantly predicted presence of all five ground rules. For I don't know, I don't understand, ignorant interviewer, and promise to tell the truth, the 4- to 6-year-olds were less likely to receive each ground rule than the 7- to 9-year-olds and the 10- to 12-year-olds. For you're wrong, the 4- to 6-year-olds were less likely to receive the you're wrong ground rule than the 7- to 9-year-olds but not the 10- to 12-year-olds.

\section{Ground Rules Practice}

Interviewers practiced an average of $2.23(S D=1.09)$ out of 3 ground rules per interview. The number of ground rules they practiced significantly differed as a function of children's age, $F(2,227)=7.24, p=.001, \eta^{2}=.06$. Post-hoc Fisher's LSD tests demonstrated that the 10 - to 12-year-olds received significantly fewer practice questions $(M=1.84, S D=1.28)$ than the 4 - to 6-year-olds $(M=2.33, S D=0.92, p=.01)$ and the 7- to 9-year-olds $(M=2.49, S D=0.98 ; p<$ $.001)$. The two younger age groups did not significantly differ from one another $(p=.34)$.

We then conducted follow-up analyses to examine the age effect across the three ground rules. We conducted separate logistic regressions with age as the predictor (using the youngest age as the comparison group) and the practice of each ground rule as the criterion. Full results are presented in Table 1 and Figure 1. Age significantly predicted practice of all three ground rules. For all three ground rules, the 10- to 12 -year-olds were less likely to receive practice questions than the 4- to 6-year-olds. The two younger age groups did not differ. 


\section{Ground Rules Performance}

Children were highly accurate $(93 \%)$ in responding to practice questions. Their overall accuracy significantly differed as a function of children's age, $F(2,195)=6.22, p=.002, \eta^{2}=$ .06. Post-hoc Fisher's LSD tests demonstrated that the 4- to 6-year-olds were significantly less accurate $(87 \%)$ than the 7 - to 9 -year-olds $(95 \%, p=.01)$ and the 10 - to 12 -year-olds $(97 \%, p=$ .002). The two older age groups did not differ from one another $(p=.61)$.

We then conducted follow-up analyses to examine the age effect across the three ground rules. We conducted separate logistic regressions with age as the predictor (using the youngest age as the comparison group) and the accuracy to each ground rule as the criterion. Full results are presented in Table 1 and Figure 1. Age significantly predicted accuracy to all three ground rule practice questions. For I don't know, the 4- to 6-year olds were significantly less accurate than the 10- to 12-year-olds but not the 7- to 9-year-olds. For I don't understand, none of the pairwise comparisons were significantly different. For you're wrong, the 4- to 6-year-olds were significantly less accurate than the 7- to 9-year-olds but not the 10- to 12-year-olds.

\section{Discussion}

Overall, our results demonstrated that highly-trained interviewers generally followed recommendations by presenting and practicing ground rules with children. However, we observed an important systematic adjustment in ground rules administration. Interviewers' presentation and practice of ground rules significantly differed by age, such that interviewers were less likely to present but more likely to practice the ground rules with younger children compared to older children. Given that these interviews were conducted prior to the most recent NCAC recommendations (which suggested adaptations or omissions to ground rules for young children), this pattern of results suggest that interviewers have long held some beliefs regarding 
the efficacy of ground rules with young children. Nevertheless, our study could not capture interviewers' true motivation for administering ground rules at different rates to differently-aged children. Thus, more research is necessary to fully understand the reasons interviewers omit the ground rules with certain children.

It is possible that interviewers believe that younger children struggle with understanding and applying ground rules, and as a result they either take extra care to practice the ground rules with younger children or see them as a waste of time and omit them altogether. This idea is also reflected in a recent review paper which concluded that preschoolers may lack key cognitive abilities (e.g., metacognition) that are necessary to properly apply ground rules and argued that they then take up valuable time by heightening the risk of premature exhaustion for children with a limited attention span (Cordisco Steele, 2015). Although the positive effects of ground rules do vary as a function of children's age (Brubacher et al., 2015), it is premature to conclude that there are no benefits to the administration of ground rules with young children or that the risks outweigh the benefits. Instead, empirical examinations should first test whether ground rules may have the proposed negative effects (e.g., exhaustion) that guide these new recommendations. Recent laboratory findings revealed that administration of ground rules in laboratory settings typically lasts around two minutes (Dickinson et al., 2015). Future research should examine whether this is the typical length of ground rules administration in real-world interviews and if that amount of time increases the risk of premature interview fatigue in younger children.

Additionally, our results revealed that children were fairly accurate in responding to practice questions. Although there were significant age differences, even the youngest children provided correct responses over $80 \%$ of the time. These results are consistent with laboratory findings suggesting that even 4-year-old children can accurately respond to most practice 
questions (Dickinson et al., 2015). However, laboratory findings also show that children may struggle more with the I don't understand practice questions and this pattern was not reflected in our results. The reason for this discrepancy is unclear. It is possible that certain practice questions within a specific ground rule (e.g., "What is your gender" versus "What is your ocular pigmentation" for I don't understand) are more or less difficult for children which leads to different results within those categories. Nevertheless, the high rates of accuracy across the practice questions likely reflect that children do initially understand the concepts and can immediately apply them which provides some support for continued administration.

\section{Limitations}

The present study provides insight into the current practices of ground rules administration, but it is important to consider the limitations of our methodology when interpreting the results and their practical implications. The first limitation to consider is the representativeness of our sample. The interviewers in our sample were highly trained and worked in child advocacy centers in the United States that regularly take part in trainings and collaborations with one of the authors. The interviewers also had to agree to allow us to review their transcripts which poses a risk for sampling bias. Therefore, the presentation and practice of ground rules by interviewers in our sample may not generalize to other jurisdictions or other interviewers. Despite this, our results demonstrate how highly trained interviewers apply their training and the conditions under which they may deviate from the recommended practices. Additionally, we were not able to obtain some types of demographic information (e.g., language ability, cultural differences) about the children in our sample. It is possible that some of the interviewers' practices and children's performance are attributable to individual differences beyond age that we were not able to observe with the available data. 
Second, we did not have information regarding when these interviews took place in relation to when interviewers received their training. Interviewers are more likely to adhere to recommendations when interviews take place shortly after training unless they engage in prolonged training containing regular peer review and/or evaluation (Benson \& Powell, 2015; Stolzenberg \& Lyon, 2015). Therefore, variation (e.g., omission of certain ground rules) may reflect differences in the amount of time that has elapsed since participation in training.

Third, we only assessed children's first trial for practice questions. It is possible that although younger children were less accurate on the first trial, they reached accuracy levels of the older children after additional trials. This potential increase in accuracy would come at the cost of an increase in time spent reviewing the ground rules which has been cited as a concern for administration (Cordisco Steele, 2015). Thus, future research should determine whether it takes substantial time for younger children to correctly respond to practice questions.

\section{Conclusion}

The present study demonstrates that highly trained interviewers generally follow best practices regarding ground rules administration but they do make systematic adjustments by either omitting the ground rules entirely or practicing them more often with younger children compared to older children. These changes are important as they reflect the practices of interviewers in the real-world that have yet to be examined in empirical research. This knowledge about what interviewers are doing should be reflected in future studies of ground rules which will increase the applicability of research findings to practice. Research on these specific deviations can guide future forensic practice by showing that they should be adjusted or maintained depending on whether they are helpful, harmful, or have no effect on interviews. 
We encourage other researchers to contribute to the small but growing literature on ground rules. Future research should examine the incremental and combined impact of the ground rules, as well as whether the time it takes to complete the ground rules comes at a significant cost for young children. Finally, before making any substantial changes, we must consider "benefits" beyond children's responses. It is possible that there are benefits to ground rules that we have yet to measure or even consider (e.g., effect of giving interviewers a structured introduction). Ground rules are a key component to most interviewing guidelines and therefore warrant more scholarly attention. 


\section{References}

Ahern, E. C., Stolzenberg, S. N., \& Lyon, T. D. (2015). Do prosecutors use interview instructions or build rapport with child witnesses? Behavioral Sciences and the Law, 33, 476-492. http://dx.doi.org/10.1002/bsl.2183

American Professional Society on the Abuse of Children [APSAC] Taskforce (2012). Practice guidelines: Forensic interviewing in cases of suspected child abuse. Retrieved on June 24, 2019 from https://www.apsac.org/guidelines.

Benson, M. S. \& Powell, M. B. (2015). Evaluation of a comprehensive interactive training system for investigative interviewing of children. Psychology, Public Policy, and Law, 21(3), 309-322. http://dx.doi.org/10.1037/law0000052

Brubacher, S. P., Poole, D. A., \& Dickinson, J. J. (2015). The use of ground rules in investigative interviews with children: A synthesis and call for research. Developmental Review, 36, 15-33. http://dx.doi.org/10.1016/j.dr.2015.01.001

Cordisco Steele, L., \& National Children's Advocacy Center (2015). Do forensic interview protocols work for preschoolers? Huntsville, AL: National Children's Advocacy Center.

Danby, M. C., Brubacher, S. P., Sharman, S. J., \& Powell, M.B. (2015). The effects of practice on children's ability to apply ground rules in a narrative interview. Behavioral Sciences and the Law, 33(4), 446-458. http://dx.doi.org/10.1002/bs1.2194.

Dickinson, J. J., Brubacher, S. P., \& Poole, D. A. (2015). Children's performance on ground rules questions: Implications for forensic interviewing. Law and Human Behavior, 39(1), 87-97. http://dx.doi.org/10.1037/lhb0000119. 
Earhart, B., Brubacher, S. P., Powell, M. B., Westera, N.J., \& Goodman-Delahunty, J. (2017). Judges' delivery of ground rules to child witnesses in Australian courts. Child Abuse and Neglect, 74, 62-72. http://dx.doi.org/10.1016/j.chiabu.2017.08.005

Earhart, B., La Rooy, D. J., Brubacher, S. P., \& Lamb, M. E. (2014). An examination of “don't know" responses in forensic interviews with children. Behavioral Sciences \& the Law, 32(6), 746-761. http://dx.doi.org/10.1002/bsl.2141

Lyon, T.D. (2014). Interviewing children. Annual Review of Law \& Social Science, 10, 73-89. National Children's Advocacy Center. (2019). National Children's Advocacy Center's Child Forensic Interview Structure. Huntsville, AL: Author.

Orbach, Y., Hershkowitz, I., Lamb, M. E., Esplin, P. W., \& Horowitz, D. (2000). Assessing the value of structured protocols for forensic interviews of alleged child abuse victims. Child Abuse \& Neglect, 24, 733-752. http://dx.doi.org/10.1016/S0145-2134(00)00137-X

Stolzenberg, S. N. \& Lyon, T. D. (2015). Repeated self- and peer-review leads to continuous improvement in child interviewing performance. Journal of Forensic Social Work, 5(1-3), 20-28. http://dx.doi.org/10.1080/1936928X.2015.1068721

Teoh, Y.S., \& Lamb, M.E. (2010). Preparing children for investigative interviews: Rapport building, instruction, and evaluation. Applied Developmental Science, 14, 154-163. http://dx.doi.org/10.1080/10888691.2010.494463

Waterman, A. H. \& Blades, M. (2011). Helping children correctly say "I don’t know" to unanswerable questions. Journal of Experimental Psychology: Applied, 17, 396-405. http://dx.doi.org/10.1037/a0026150 
Table 1. Full results from logistic regressions with age group as the predictor and presentation/practice/performance on each ground rule as the criterion.

\begin{tabular}{|c|c|c|c|c|c|}
\hline Measure & Ground Rule & Omnibus Analysis & Age & Prevalence & Pairwise Comparisons \\
\hline \multirow{15}{*}{ Presentation } & \multirow{3}{*}{ I don't know } & \multirow{3}{*}{ Wald $\chi^{2}(2)=13.88, p=.001$} & $4-6$ & $75 \%$ & -- \\
\hline & & & $7-9$ & $92 \%$ & Wald $\chi^{2}(1)=7.80, p=.01$ \\
\hline & & & $10-12$ & $93 \%$ & Wald $\chi^{2}(1)=8.14, p=.004$ \\
\hline & \multirow{3}{*}{ I don't understand } & \multirow{3}{*}{ Wald $\chi^{2}(2)=21.27, \mathrm{p}<.001$} & $4-6$ & $62 \%$ & -- \\
\hline & & & $7-9$ & $84 \%$ & Wald $\chi^{2}(1)=9.37, p=.002$ \\
\hline & & & $10-12$ & $90 \%$ & Wald $\chi^{2}(1)=15.11, p<.001$ \\
\hline & \multirow{3}{*}{ You're wrong } & \multirow{3}{*}{ Wald $\chi^{2}(2)=12.60, p=.002$} & $4-6$ & $80 \%$ & -- \\
\hline & & & $7-9$ & $96 \%$ & Wald $\chi^{2}(1)=8.31, p=.004$ \\
\hline & & & $10-12$ & $82 \%$ & Wald $\chi^{2}(1)=0.15, p=.70$ \\
\hline & \multirow{3}{*}{ Ignorant interviewer } & \multirow{3}{*}{ Wald $\chi^{2}(2)=14.38, p=.001$} & $4-6$ & $45 \%$ & -- \\
\hline & & & $7-9$ & $70 \%$ & Wald $\chi^{2}(1)=10.12, p=.001$ \\
\hline & & & $10-12$ & $70 \%$ & Wald $\chi^{2}(1)=9.86, p=.002$ \\
\hline & \multirow{3}{*}{$\begin{array}{l}\text { Promise to tell the } \\
\text { truth }\end{array}$} & \multirow{3}{*}{ Wald $\chi^{2}(2)=18.78, \mathrm{p}<.001$} & $4-6$ & $80 \%$ & -- \\
\hline & & & $7-9$ & $94 \%$ & Wald $\chi^{2}(1)=6.18, p=.01$ \\
\hline & & & $10-12$ & $99 \%$ & Wald $\chi^{2}(1)=7.79, p=.01$ \\
\hline \multirow{9}{*}{ Practice } & \multirow{3}{*}{ I don't know } & \multirow{3}{*}{ Wald $\chi^{2}(2)=15.40, \mathrm{p}<.001$} & $4-6$ & $93 \%$ & -- \\
\hline & & & $7-9$ & $86 \%$ & Wald $\chi^{2}(1)=1.52, p=.22$ \\
\hline & & & $10-12$ & $68 \%$ & Wald $\chi^{2}(1)=11.40, p=.001$ \\
\hline & \multirow{3}{*}{ I don't understand } & \multirow{3}{*}{ Wald $\chi^{2}(2)=31.09, \mathrm{p}<.001$} & $4-6$ & $96 \%$ & -- \\
\hline & & & $7-9$ & $94 \%$ & Wald $\chi^{2}(1)=0.39, p=.53$ \\
\hline & & & $10-12$ & $64 \%$ & Wald $\chi^{2}(1)=12.82, p<.001$ \\
\hline & \multirow{3}{*}{ You're wrong } & \multirow{3}{*}{ Wald $\chi^{2}(2)=22.51, \mathrm{p}<.001$} & $4-6$ & $96 \%$ & -- \\
\hline & & & $7-9$ & $92 \%$ & Wald $\chi^{2}(1)=0.87, p=.35$ \\
\hline & & & $10-12$ & $68 \%$ & Wald $\chi^{2}(1)=13.18, p<.001$ \\
\hline \multirow{9}{*}{ Performance } & \multirow{3}{*}{ I don't know } & \multirow{3}{*}{ Wald $\chi^{2}(2)=7.99, p=.02$} & $4-6$ & $83 \%$ & -- \\
\hline & & & $7-9$ & $92 \%$ & Wald $\chi^{2}(1)=2.37, p=.12$ \\
\hline & & & $10-12$ & $98 \%$ & Wald $\chi^{2}(1)=4.48, p=.03$ \\
\hline & \multirow{3}{*}{ I don't understand } & \multirow{3}{*}{ Wald $\chi^{2}(2)=7.461, p=.02$} & $4-6$ & $91 \%$ & -- \\
\hline & & & $7-9$ & $98 \%$ & Wald $\chi^{2}(1)=2.70, p=.101$ \\
\hline & & & $10-12$ & $100 \%$ & Wald $\chi^{2}(1)=0.00, p=1.00$ \\
\hline & \multirow{3}{*}{ You're wrong } & \multirow{3}{*}{ Wald $\chi^{2}(2)=6.53, p=.04$} & $4-6$ & $86 \%$ & -- \\
\hline & & & $7-9$ & $97 \%$ & Wald $\chi^{2}(1)=4.86, p=.03$ \\
\hline & & & $10-12$ & $93 \%$ & Wald $\chi^{2}(1)=1.23, p=.27$ \\
\hline
\end{tabular}

Note. Pairwise comparisons use the youngest age group (4- to 6- year-olds) as the reference group. 


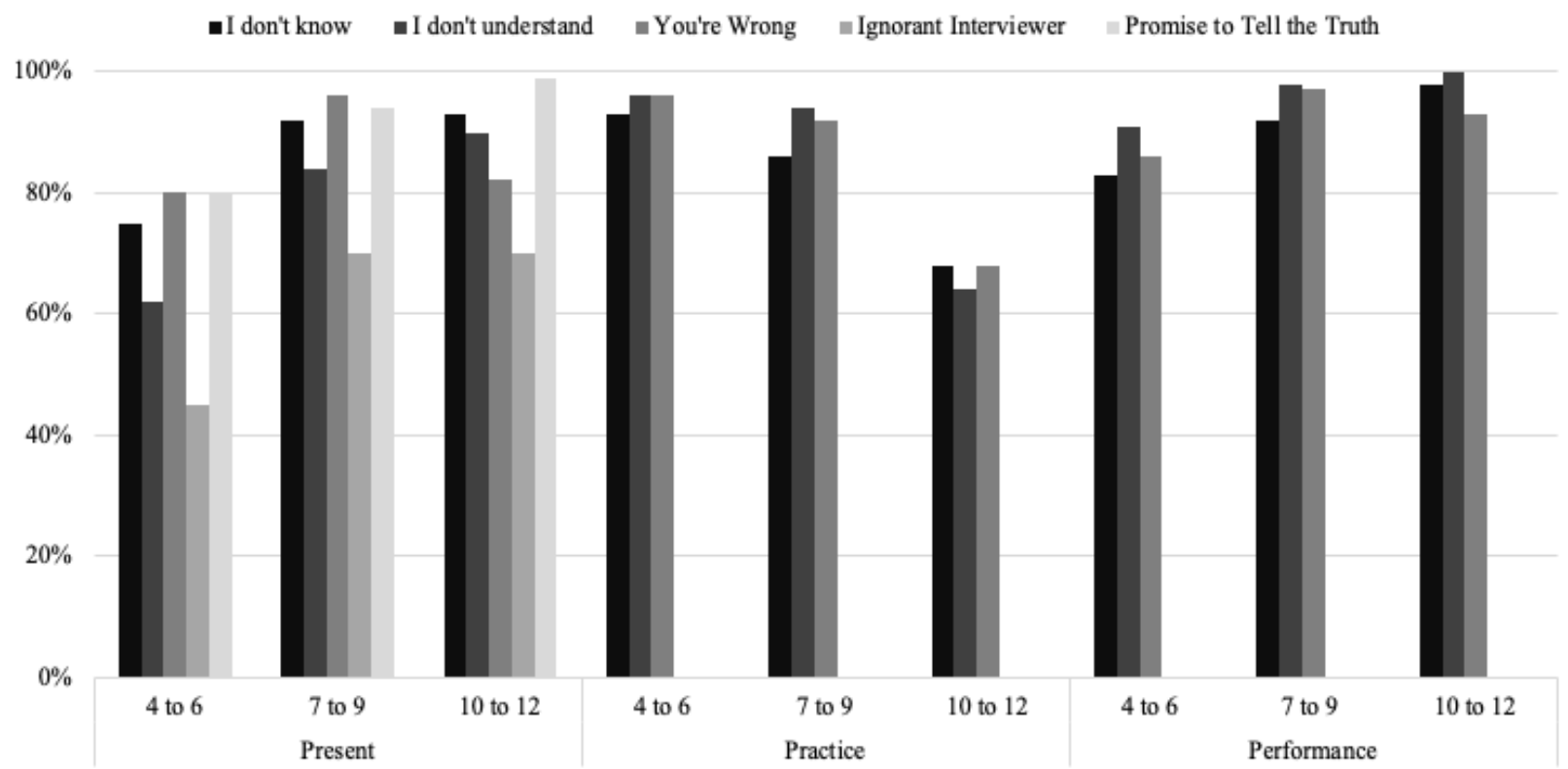

Figure 1. Percentage of presentation, practice, and accurate performance for each of the ground rules by children's age. Denominator used for presentation on all five ground rules was the full sample $(N=241)$. Denominator for practice is the number of interviews where the ground rule was present (I don't know, $n=207$; I don't understand, $n=187$; you're wrong, $n=207$ ). Denominator used for performance is the number of interviews where the ground rule was practiced (I don't know, $n=170$; I don't understand, $n=157$; you're wrong, $n=179$ ). 